\title{
Las estructuras de dativo simpatético en lengua alemana: ¿dativo, o también 'acusativo simpatético'?
}

\author{
Rafael López-Campos Bodineau \\ Profesor Titular de Universidad \\ Universidad de Sevilla \\ Facultad de Filología \\ c/Palos de la Frontera, s/n \\ 41004 Sevilla \\ rafali@us.es
}

\section{LAS ESTRUCTURAS DE DATIVO SIMPATÉTICO EN LENGUA ALE- MANA: ¿DATIVO, O TAMBIÉN 'ACUSATIVO SIMPATÉTICO'?}

RESUMEN: Con el presente trabajo se deja constancia de diversos modelos oracionales de los que nos valemos en la lengua alemana para poner de manifiesto acciones verbales vinculadas a partes del cuerpo, en el caso concreto en que esta aparece referenciada dentro del marco de un constituyente de tipo adverbial. Así, en el artículo se delimitan cuatro constantes o modelos sintácticos, dos integrados por dativo y dos por acusativo también como segundo actante, los cuales comparten características comunes, de tal modo que, partiendo del rol como 'dativo posesivo' que la gramática tradicional alemana le otorga a los dos modelos con dativo, muy especialmente al primero de ellos (NOM, AKK, ADV EST) en oraciones del tipo er klopft mir auf die Schulter, del mismo modo podríamos afirmar que los dos modelos con acusativo tendrian un carácter igualmente 'posesivo', y, así, sería posible hablar de un 'acusativo simpatético' en lengua alemana.

PALABRAS CLAVE: Sintaxis alemana; dativo alemán; acusativo alemán; dativo simpatético; dativo posesivo.

SUMARIO: 0. Punto de partida y objetivos. 1. El modelo oracional $\mathrm{V}$ [NOM, DAT, ADV DIR]. 2. El modelo oracional $\mathrm{V}$ [NOM, AKK, ADV EST]. 3. El modelo oracional $\mathrm{V}$ [NOM, DAT, ADV EST]. 4. El modelo oracional V [NOM, AKK, ADV DIR]. 5. Conclusiones
STRUCTURES OF THE SYMPATHETIC DATIVE IN GERMAN: DATIVE OR ALSO "SYMPATHETIC ACCUSATIVE"?

ABSTRACT: This work looks at different sentence patterns used in German in order to highlight verbal actions associated with parts of the body in the specific case that they are referred to within the framework of an adverbial constituent. Therefore, this article defines four constants or syntactic models, two comprised of the dative as second actant and two comprised of the accusative, also as second actant, which share common traits in such a way that, based on the role of "possessive dative" that traditional German grammar gives the two models with the dative, especially the first of these (NOM, ACC, ADV EST) in sentences such as er klopft mir auf die Schulter, we can also affirm that the two patterns with the accusative would be equally "possessive" and, therefore, it would be possible to talk about a "sympathetic accusative" in the German language.

KEYWORDS: German syntax; German dative; German accusative; sympathetic dative; possessive dative.

SUMMARY: 0 . Starting point and objectives. 1. $\mathrm{V}$ sentence model [NOM, DAT, ADV DIR]. 2. $\mathrm{V}$ sentence model [NOM, ACC, ADV EST]. 3. V sentence model [NOM, DAT, ADV EST]. 4. V sentence model [NOM, ACC, ADV DIR]. 5. Conclusions
LES STRUCTURES DE DATIF SYMPATHÉTIQUE EN LANGUE ALLEMANDE : DATIF, OU ÉGALEMENT "ACCUSATIF SYMPATHÉTIQUE "?

RÉSUMÉ: Le présent travail rend compte de différents modèles propositionnels dans lesquels la langue allemande nous permet de mettre en évidence des actions verbales liées à des parties du corps, dans le cas précis où celle-ci apparait comme étant référenciée dans le cadre d'un constituant de type adverbial. Ainsi, dans cet article, quatre constituants ou modèles syntaxiques sont délimités - deux comprenant le datif comme second actant, et deux comprenant l'accusatif également comme second actant - , partageant tous des caractéristiques communes, de telle sorte que partant du rôle de " datif possessif " que la grammaire traditionnelle allemande attribue aux deux modèles concernant le datif, et tout particulièrement au premier de ceux-ci (NOM, AKK, ADV EST) dans des phrases de type er klopft mir auf die Schulter, nous pourrions affirmer que les deux modèles concernant l'accusatif auraient également un caractère " possessif ", et il serait donc possible de parler d'un " accusatif sympathétique " en langue allemande.

MOTS-CLÉS: Syntaxe allemande ; datif allemand ; accusatif allemand ; datif sympathétique ; datif possessif.

SOMMAIRE: 0. Point de départ et objectifs. 1. Le modèle propositionnel V [NOM, DAT, ADV DIR]. 2. Le modèle propositionnel V [NOM, AKK, ADV EST]. 3. Le modèle propositionnel $\mathrm{V}$ [NOM, DAT, ADV EST]. 4. Le modèle propositionnel $\mathrm{V}$ [NOM, AKK, ADV DIR]. 5. Conclusions 


\section{Las estructuras de dativo simpatético en lengua alemana: ¿dativo, o también 'acusativo simpatético'?}

Rafael López-Campos Bodineau

\section{Punto de partida y objetivos}

Dentro del marco de los distintos tipos de dativo en lengua alemana, cuya clasificación y correspondientes marcadores semánticos fueron ampliamente estudiados por, entre otros, W.Admoni (1982), G.Drosdowski (1995), G.Helbig y J.Buscha (1996), Schäfer (2015), etc. ${ }^{1}$, cabe destacar la estabilidad actancial de la mayoría de los modelos. Por ejemplo, véase la tendencia de la mayoría de los verbos introductores de dativo de beneficio o perjuicio a quedar vinculados con la trivalencia V [NOM, AKK, DAT], en oraciones del tipo er schenkt mir das Fahrrad, o bien er stiehlt mir das Fahrrad ${ }^{2}$, respectivamente; o bien de cualquier verbo introductor de dativo objetivo o subjetivo de los tipos 'Dativ des Zustandsträgers' o 'Dativ des Maßstabs' a hacerlo con la trivalencia V [NOM, PRÄD, DAT], en oraciones del tipo die Musik ist mirzu laut, das Musikhören ist mir sehr angenehm, dieser Computer ist mir viel zu teuer, etc.

En el marco de la citada estabilidad sintáctica, y tal como ya hicimos en R.López-Campos (1993), queremos referirnos muy singularmente al denominado dativo posesivo (Dativus Possessivus como Pertinenzdativ o Dativus Sympatheticus) en cuyo entorno queda implicada alguna 'parte del cuerpo' vinculada al agente o alguno de los constituyentes de la acción —o bien alguna 'prenda de vestir': el 'Pertinenzdativ' aplica tanto a 'partes del cuerpo' como a 'prendas de vestir'

1 Es obligado referirse a los postulados acerca del 'Dativ' que recopila M.Hocke (1987: 3-54): hasta un total de 27 lingüistas del siglo XX que de forma directa o indirecta estudiaron la relación posesiva del 'Dativ', y también del 'Akkusativ' en las distintas lenguas. Por tomar algún ejemplo, W.Admoni (1982: 121-122) jerarquiza los tipos de dativo en tres niveles, atendiendo al grado de obligatoriedad que presentan: dativo obligatorio o 'notwendiges indirektes Objekt', que viene dado por la valencia obligatoria del verbo (entsprechen en es entspricht unseren Erwartungen, ähneln en er ähnelt seinem Vater, etc.); dativo opcional o 'nicht notwendiges indirektes Objekt', en cuyo entorno se situarían todos aquellos dativos cuya aparición en la oración no es obligatoria (ich öffne dir die Tür > ich öffne die Tür, etc.); y el dativo ético o 'dativus ethicus', en cuyo entorno se situarian todos aquellos dativos que ponen de manifiesto una implicación emocional por parte de alguno de los actantes de la oración (du bist mir zu schlau!, was denkst du dir dabei?). G.Helbig y J.Buscha (1996: 288), por su parte, aborda su clasificación de los tipos de dativo en torno a dos grandes grupos: dativo como objeto de un verbo o 'Objekt zum Verb' (er gibt dem Freund ein Buch), y dativo como elemento secundario o 'sekundäres Satzglied'; y dentro de esta segunda categoría establece una detallada subcategorización en dativo del tipo 'Pertinenzdativ' referido siempre a partes del cuerpo (wir waschen uns die Hände), dativo del tipo 'Träger-Dativ' referido siempre a prendas de vestir (er zieht ihr den Mantel an), dativo de beneficio o 'Dativus commodi' (ich kaufe mir einen neuen Atlas), dativo de perjuicio o 'Dativus incommodi' (das Kind zerbrach den Eltern die Vase), dativo del tipo 'Dativ des Zustandsträgers' referido a apreciaciones personales de tipo objetivo (dieser Erfolg ist ihm eine Freude), dativo del tipo 'Dativ des Maßstabs' referido siempre a apreciaciones personales de tipo subjetivo (er arbeitet mir zu langsam), dativo ético o 'ethischer Dativ' (falle mir nicht!) y aposición o 'Apposition' (der Lehrer antwortet Herrn Müller, dem Direktor der Schule).

2 Partimos del concepto apriorístico de valencia, tal como fue originariamente propugnado por G. Helbig y W. Schenkel (1969) o U. Engel y H. Schmacher (1976), etc., en virtud del cual a un verbo cualquiera le es asignada una valencia determinada dependiendo no del plano oracional que inferimos del contexto en que es empleado sino de la distribución de actantes a la que es vinculado de forma prototípica. 
(J.Schmid, 2006: 955)—; y muy singularmente queremos centrarnos en aquellos casos de 'Pertinenzdativ' en que la 'parte del cuerpo' constituye el núcleo de la función ADV DIR a partir de una estructura base del tipo V [NOM, DAT, ADV DIR]. ${ }^{3}$ Partamos de la siguiente oración, cuya distribución de constituyentes resulta paradigmática dentro del dativo posesivo al que hemos hecho referencia y, más concretamente, de la citada estructura de partida: er klopft ihm auf die Schulter.

Si atendemos no tanto a la función sintáctica de los constituyentes (NOM, DAT, ADV DIR) como a la forma propiamente dicha de determinante más sustantivo que presenta tanto el 'segundo' constituyente DAT (mir) como el 'tercero' ADV DIR (die Schulter), constatamos que en dicha presentación formal - de determinante más sustantivo, como decimos- también podrían caber otras distribuciones de caso resultantes de combinar las funciones DAT y AKK en esos mismos constituyentes: en el 'segundo' a través del régimen directo que marca la base verbal, y en el 'tercero' a través del caso gramatical ('Dativ' o 'Akkusativ') que introduce la preposición según se trate de una relación dinámica (ADV DIR) o estática (ADV EST). De este modo surgirian cuatro combinaciones diferentes:

V [NOM, DAT, ADV DIR] er klopft ihm ${ }_{\text {[DAT] }}$ auf die Schulter ${ }_{\text {[ADV DIR: AKK] }}$
V [NOM, AKK, ADV EST] er nimmt mich ${ }_{[\text {AKK] }}$ an der Hand ${ }_{\text {[ADV EST: DAT] }}$
V [NOM, DAT, ADV EST] das Schampoo brennt mir ${ }_{\text {[DAT] }}$ in den Augen ${ }_{\text {[ADV EST: DAT] }}$
V [NOM, AKK, ADV DIR] er nimmt mich ${ }_{[\mathrm{AKK}]}$ in die Arme ${ }_{\text {[ADV DIR: AKK] }}$

A partir de un análisis de esos cuatro modelos, nos proponemos 1) valorar en qué medida es posible alterar las estructuras que emanan de cada base verbal o si, por el contrario, se trata de modelos inamov-

3 Véase la categorización propugnada por G.Helbig y J.Buscha (1996: 289), C. Mellado (2006: 186) o C. Mellado (2012) que prevé tres modelos diferentes según la función sintáctica en cuyo entorno es situado el elemento oracional denotativo de la "parte del cuerpo'. El primero de ellos, prototípicamente bivalente, está basado en una estructura oracional del tipo V [NOM, DAT] a partir de bases como schmerzen (meinem Vater schmerzt der Kopf), wehtun (meinem Vater tut der Kopf weh) o jucken (mir juckt der Kopf) en cuyo entorno la 'parte del cuerpo' ocupa la función NOM. El segundo de ellos, trivalente, responde a V [NOM, AKK, DAT] a partir de bases como waschen (ich wasche mir die Hände) o kratzen (ich kratze mir den Bart), y en todos ellos la 'parte del cuerpo' ocupa la función AKK. Y el tercero, también trivalente, responde a V [NOM, DAT, ADV DIR] a partir de bases como klopfen (er klopft mir auf die Schulter) o gehen (das geht mir auf die Nerven) y en todos ellos la 'parte del cuerpo' constituye el núcleo de la función ADV DIR. Tal como indicábamos anteriormente en alusión a SCHMID (2006: 955), el concepto Pertinezdativ afecta igualmente a acciones relacionadas con 'partes del cuerpo' y 'prendas de vestir'. Así, las tres variables constatables en el marco del dativo posesivo podrian ser igualmente aplicables a la antedicha variante del tipo 'Träger-Dativ', en cuyo entorno queda implicada una prenda de vestir; a saber: disponemos de una estructura oracional del tipo V [NOM, DAT] en aquellos casos en que la 'prenda de vestir' ocupa la función NOM (véase rutschen en dem Jungen rutscht die Hose), V [NOM, AKK, DAT] en caso de que ocupe la función AKK (véase anziehen en er zieht ihr den Mantel an, o rücken en der Besucher rückt sich den Schlips zurecht) —en este caso el constituyente DAT podría mostrar carácter optativo: er zieht sich den Mantel an > er zieht den Mantel an, etc., aunque en el caso de las 'partes del cuerpo' esta versatilidad resultará mayor o menor según el significado del verbo propiamente dicho: compárese er putzt sich die Zähne > er putzt die Zähne con er wäscht sich die Hände > ? er wäscht die Hände-, o $\mathrm{V}$ [NOM, DAT, ADV DIR] en caso de que la 'prenda de vestir' constituya el núcleo de la función ADV DIR (véase treten en ich trete ihm auf den Schuh o fallen en dem Kind fällt die Mütze vom Kopf). Nuestro análisis se restringe a la tercera de las opciones, es decir, a aquellos casos en que la 'parte del cuerpo' constituye el núclo de la función ADV DIR. 
ibles, y 2) determinar en qué medida cada uno de esos cuatro modelos es portador de una carga o valor 'posesivo' similar al atribuible a nuestra oración de partida er klopft ihm auf die Schulter.

\section{El modelo oracional V [NOM, DAT, ADV DIR]}

Comencemos por el modelo vinculado al ejemplo que hemos tomado como punto de partida, el modelo V [NOM, DAT, ADV DIR], y, para ello, partamos de un pequeño corpus integrado por las siguientes tres oraciones - véase según orden alfabético de la base verbal-: ${ }^{4}$

(01) klopfen > er klopft ihm auf die Schulter

(02) sehen > er sieht mir in die Augen

(03) treten > ich trete ihm auf den Schuh

Si analizamos la dependencia de los constituyentes que integran estas tres oraciones respecto de sus correspondientes bases verbales, tanto en estos casos como en cualesquiera otros que pudiéramos mencionar (der Regen tropft mir auf den Kopf, er legt mir die Hand auf die Schulter, er flüstert mir etwas ins Ohr, sie lacht mir ins Gesicht, das treibt ihm Tränen in die Augen, er lügt mir ins Gesicht, etc.), verificamos las siguientes cuatro constantes sintácticas: rigidez formal de caso en sus tres constituyentes, limitación formal del constituyente ADV DIR, competencia respecto de las formas 'Genitivattribut' o 'adjektivisches Attribut' y carácter obligatorio del segundo constituyente (DAT).

En primer lugar, cualquiera de estos ejemplos muestra una clara rigidez formal en sus tres constituyentes; no solo del primero (NOM), sino también del segundo (DAT) y del tercero (ADV DIR). Véase, así, la imposibilidad de alterar el segundo constituyente de DAT a AKK, o bien, en el caso del tercero, la imposibilidad de alterar el régimen preposicional del 'Akkusativ' propio de la relación ADV DIR a 'Dativ':

(01a) er klopft ihm auf die Schulter

(01b) *er klopft ihn auf die Schulter

(01c) *er klopft ihm auf der Schulter

Y del mismo modo podemos proceder con (02) o (03): er sieht mir in die Augen > *er sieht mich in die Augen > *er sieht mir in den Augen, o bien ich trete ihm auf den Schuh > *ich trete ihn auf den Schuh > *ich trete ihm auf dem Schuh, etc.

En segundo lugar, las limitaciones relativas a la morfología del constituyente ADV DIR afectan no solo al caso gramatical propiamente dicho sino a la categoría léxica, pues, en todos estos casos, la habitual triple forma adverbial de 1) grupo preposicional con marcador direccional en 'Akkusativ', 2) adverbio propiamente dicho o 3) adverbio pro-

4 Cabe destacar la compatibilidad que muestra este dativo posesivo con, dependiendo del contexto, otros posibles dativos, singularmente el dativo de beneficio (Dativus Commodi) o el dativo de perjuicio (Dativus Incommodi). Por ejemplo, la expresión (03) er tritt ihm auf den Schuh pone de manifiesto la relación propia no solo de un dativo posesivo sino también de un dativo de perjuicio en la medida en que la acción resultante constituye, en principio, un claro perjuicio para la persona destinataria de la acción. 
nominal del tipo darauf, darüber, etc. —véanse, a partir de un verbo adlativo como fliegen, las correspondencias er fliegt auf die Insel > er fliegt dorthin, o bien, a partir de un verbo igualmente adlativo como schreiben, las correspondencias er schreibt auf das Papier > er schreibt darauf -, queda muy limitada a la primera de las tres opciones. Véase:

(01a) er klopft ihm auf die Schulter

(01d) *er klopft ihm dorthin, dahin, etc.

(01e) *er klopft ihm darauf

Y exactamente del mismo modo en el caso de (02) o (03): sie sieht mir in die Augen > *er sieht mir dorthin > (02e) *er sieht mir darin, o bien ich trete ihm auf den Schuh > *ich trete ihm dorthin > *ich trete ihm darauf, etc.

En tercer lugar, en todos estos casos es posible establecer un correlato o competencia de uso respecto de las formas 'Genitivattribut' o 'adjektivisches Attribut'. ${ }^{6}$ Véase:

(01a) er klopft ihm auf die Schulter

(01f) er klopft auf seine Schulter, er klopf auf die Schulter seines Nachbarn

Y exactamente del mismo modo sie sieht mir in die Augen > er sieht in meine Augen, o bien er tritt mir auf den Schuh > er tritt auf meinen Schuh, etc. La opción (01f), y correspondiente en el resto de verbos seleccionados, se presenta como correlato semántico de (01a), ello sin detrimento de las alteraciones pragmalingüísticas relacionadas con una focalización de la atención en (01f) no tanto en la acción verbal como en la información contenida en el grupo nominal del constituyente ADV DIR; bien en el valor de su componente nominal propiamente dicho ('Genitivattribut': er klopft auf die Schulter seines Nachbarn), o bien en la relación de pertenencia puesta de manifiesto por el posesivo en sí ('adjektivisches Attribut': er klopft auf seine Schulter) - en este caso, merced a esas alteraciones, podría marcarse de la siguiente manera: (01f) ${ }^{(i)}$ er klopft auf seine Schulter, (01f) (i) er klopft auf die Schulter seines Nachbarn-. En relación con posibles competencias de uso entre las fórmulas de dativo propiamente dicho más determinante posesivo y posibles matices diferenciadores entre uno y otro, véase el recorrido histórico de

5 En algún caso seria posible aplicar un adverbio pronominal del tipo daraufo drauf en contextos relativamente coloquiales del tipo 'er klopft ihm auf die Schulter drauf en que el constituyente drauf en cualquier caso no prescinde de la relación auf die Schulter y por tanto se presenta como duplicación funcional similar a la que se produce en otros muchos verbos de posicionamiento estático: 'das Buch liegt auf dem Tisch drauf, etc.

6 Efectivamente, y tal como igualmente establecen G.Helbig y J.Buscha (1996: 291), en el caso tanto del Dativus Possessivus como del Träger-Dativ es posible establecer no identidad pero sí afinidad semántica entre el uso del 'Dativ' y las formas 'Genitivattribut' o 'adjektivisches Attribut'. Véanse todos aquellos casos en que la parte del cuerpo o prenda afectada ocupa la función NOM (meinem Vater schmerzt der Kopf > sein Kopf schmerzt > der Kopf meines Vaters schmerzt, etc., o bien dem Jungen rutscht die Hose > Seine Hose rutscht > die Hose des Jungen rutscht, etc.), o bien la función AKK (ich wasche mir die Hände > ich wasche meine Hände, er zieht ihr den Mantel an > er zieht ihren Mantel an, etc.), o bien aparece en el marco del grupo preposicional (er sieht mir in die Augen > er sieht in meine Augen, ich trete ihm auf den Schuh > ich trete auf seinen Schuh, etc.). 
tipo lingüístico-comparado recopilado por C. Mellado (2006).

Las constataciones establecidas en torno a la posibilidad de llevar a cabo pruebas de sustitución respecto de algún determinante posesivo, según resulta de comparar (01a) y (01f), sin embargo, no son aplicables a expresiones con carácter fraseolexemático del tipo sich ins eigene Knie schießen, etwas geht jdm. auf die Nerven, jdm. in die Hände fallen, jdm. in die Arme fallen, jdm. ins Gesicht lachen, jdm. an die Seele gehen, jdm. auf den Magen gehen o jdm. auf den Magen schlagen, jdm. auf die Nieren gehen, jdm. auf den Wecker gehen, jdm. durch Mark und Bein gehen, jdm. unter die Haut gehen, etc. Cualquiera de ellas, pese a responder igualmente al modelo establecido V [NOM, DAT, ADV DIR], no obstante, merced al citado carácter fraseolexemático y consiguiente inalterabilidad de sus partes, muestra mayores limitaciones al respecto (C. Mellado, 2006), no solo en lo relativo a la posibilidad de dotar al constituyente ADV DIR de alguna forma alternativa de adverbio o adverbio pronominal, algo que ya ocurría en el caso de las unidades 'no fraseolexemáticas' según pudimos ver en $(01 \mathrm{~d})$ o $(01 \mathrm{e})$, sino, además en este caso, en lo relativo a la posibilidad de establecer competencias de uso o correlatos respecto de la fórmula de determinante posesivo. Compárese el par (01a)-(01f) con cualquiera de estas otras unidades, por ejemplo etwas geht jdm. auf die Nerven: ${ }^{7}$

er klopft ihm auf die Schulter er klopft auf seine Schulter

die Nachbarn gehen mir auf die Nerven [fraseolexema] *die Nachbarn gehen auf meine Nerven [fraseolexema]

Finalmente, en cuarto lugar, debemos referirnos al carácter obligatorio del segundo constituyente (DAT); esto es: el constituyente DAT podría ser omisible en el marco de cualquier otra relación 'no posesiva' (er klopft auf den Tisch > erklopft), pero no en el caso de mediar 'relación posesiva', pues en ese caso su supresión resultaria agramatical (P. Polenz, 1969). Por su parte, el tercer constituyente resulta obligatorio sin restricciones. Véase:

(01a) er klopft ihm auf die Schulter

(01g) 'er klopft auf die Schulter'

(01h) *er klopft ihm

7 Los ejemplos fraseológicos que hemos indicado están vinculados a relaciones de dativo posesivo propiamente dicho, en los que está implicada una 'parte del cuerpo' (Nerven en jemand geht mir auf die Nerven, Hände en etwas fällt mir in die Hände, etc.), pero la excepcionalidad de que son exponentes sería igualmente aplicable a otras estructuras igualmente fraseológicas no necesariamente vinculadas a ninguna 'parte del cuerpo': jdm. über den Weg laufen (er läuft mir über den Weg > *er läuft über meinen Weg), jdm. auf die Schliche kommen (er kommt mir auf die Schliche > *er kommt auf meine Schliche), jdm. auf die Spur kommen (er kommt mir auf die Spur > *er kommt auf meine Spur), etc. 8 Únicamente, (01g) y equivalentes en (02) y (03) podrían mostrar gramaticalidad en caso de referencia a algún 'Genitivobjekt' implicito: er klopft auf die Schulter (des Kranken, des bewusstlosen Mädchens, etc.), er sieht in die Augen (der Leute), er tritt auf den Schuh (seines Kollegen), etc., pero, en sí mismo, sin referencia implícita a algún sustantivo de estas características, la construcción resultaría agramatical o, cuando menos, dificilmente aplicable. 
Y exactamente del mismo modo en relación con (02) o (03): sie sieht mir in die Augen > *er sieht in die Augen > * sie sieht mir, o bien er tritt mir auf den Fuß > *er tritt auf den Fuß > *er tritt mir, etc.

\section{El modelo oracional V [NOM, AKK, ADV EST]}

A partir del análisis realizado, y circunscribiendo nuestro estudio a aquellos casos en que se constata una relación 'posesiva', es decir, en el marco de una relación que, por la propia naturaleza del significado de que son exponentes las bases afectadas, involucra necesaria o al menos prototipicamente a alguna "parte del cuerpo', podemos constatar la existencia de un segundo modelo en relación con el cual, en un plano estrictamente formal, las funciones segunda y tercera -dejamos NOM al margen - intercambiarian su morfología. Así, en el marco de este segundo modelo, el verbo activa una valencia integrada, además de por el sujeto, por un grupo nominal AKK y no por uno DAT como ocurría en el primer modelo, más un grupo preposicional en esta ocasión no dinámico ADV DIR sino estático ADV EST; básicamente: V [NOM, AKK, ADV EST].

Partamos también en este caso de un pequeño corpus marcado por los siguientes tres verbos —una vez más, según orden alfabético de la base verbal-:

(04) kratzen > ich kratze sie auf dem Rücken

(05) nehmen > er nimmt mich an der Hand

(06) packen > die Polizei packte ihn an der Kehle

Al igual que en el modelo anterior, si analizamos la dependencia de los constituyentes que integran estas tres oraciones respecto de sus correspondientes bases verbales ${ }^{9}$, tanto en estos casos como en cualesquiera otros que pudiéramos mencionar (jdn. am Hals würgen, jdn. an der Hand küssen, jdn. am Arm festhalten, jdn. am Arm drücken, jdn. am Arm streicheln, jdn. am Arm verletzen, etc.), advertimos nuevamente las cuatro constantes sintácticas ya enunciadas en el caso del primer modelo: rigidez formal de caso en sus tres constituyentes, limitación formal del constituyente adverbial —en esta ocasión se trata del constituyente ADV EST-, competencia respecto de las formas 'Genitivattribut' o 'adjektivisches Attribut' y carácter obligatorio del

\footnotetext{
9 En el marco del modelo V [NOM, DAT, ADV DIR] constatábamos la existencia de muestrario relacionado tanto con 'prendas de vestir' (Dativus Possessivus) como con 'partes del cuerpo' (Träger-Dativ), y la asignación de cualquiera de las dos variantes venía dada no tanto por la implicación de una 'prenda de vestir' o 'parte del cuerpo' como elemento prototípico como por el significado de los verbos implicados que, por el valor de que son exponentes, requieren la implicación de una 'prenda de vestir' o una 'parte del cuerpo'. En el caso de V [NOM, AKK, ADV EST], sin embargo, los ejemplos mencionados (kratzen, nehmen y packen) quedan vinculados únicamente con 'partes del cuerpo', no con 'prendas de vestir'. Ciertamente, disponemos de muestrario al que podemos vincular 'prendas de vestir', por ejemplo packen (er packt mich am Pullover) o nehmen (er nimmt mich am Hemd), pero la aparición del constituyente 'prenda de vestir' no constituye un régimen prototipico del modo que ocurría en los casos de Träger-Dativ, en la medida en que el régimen ADV [EST] puede venir igualmente dado por una 'parte del cuerpo', o bien por un objeto cualquiera. Véase er packt mich am Pullover > er packt mich am Arm > er packt die Blumen am Stiel, etc.
} 
segundo constituyente —en esta ocasión AKK-. ${ }^{10}$

En primer lugar, cualquiera de estos ejemplos muestra una clara rigidez formal en sus tres constituyentes; no solo del primero (NOM), sino también del segundo (AKK) y del tercero (ADV EST). Véase, así, la imposibilidad de alterar el segundo constituyente de AKK a DAT, o bien, en el caso del tercero, la imposibilidad de alterar el régimen preposicional del 'Dativ' propio de la relación ADV EST a 'Akkusativ':

(04a) ich kratze sie auf dem Rücken

(04b) *ich kratze ihr auf dem Rücken

(04c) *ich kratze sie auf den Rücken

Y del mismo modo podemos proceder con (05) o (06): er nimmt mich an der Hand $>{ }^{*}$ er nimmt mir an der Hand $>{ }^{*}$ er nimmt mich an die Hand, die Polizei packte ihn an der Kehle $>$ *die Polizei packte ihm an der Kehle $>$ *die Polizei packte ihn an die Kehle, etc. Es decir, del mismo modo que en el modelo V [NOM, DAT, ADV DIR] el segundo constituyente, en caso de aplicarse, había de ser necesariamente DAT sin posibilidad de mutar a AKK — véase (01b) y subsiguientes-, ahora en este modelo V [NOM, AKK, ADV EST] la forma de dicho segundo constituyente, en caso de aplicarse, será necesariamente AKK sin posibilidad de mutar a DAT; y del mismo modo que en el modelo V [NOM, DAT, ADV DIR] el tercer constituyente, en caso de aplicarse, había de ser necesariamente ADV DIR - véase (01c) y subsiguientes-, ahora en este modelo $\mathrm{V}$ [NOM, AKK, ADV EST] la forma de dicho tercer constituyente, en caso de aplicarse, será necesariamente ADV EST.

En segundo lugar, una vez más, las limitaciones relativas a la morfología del constituyente ADV EST afectan no solo al caso gramatical propiamente dicho sino a la categoría léxica, pues, en todos estos casos, la habitual triple forma adverbial de 1) grupo preposicional con marcador estático en 'Dativ', 2) adverbio propiamente dicho o 3) adverbio pronominal del tipo darauf, darüber, etc. - véase, a partir de un verbo inlativo como sitzen, las correspondencias er sitzt auf dem Stuhl > er sitzt dort, o bien, a partir de ese mismo verbo, las correspondencias er sitzt auf dem Stuhl > er sitzt darauf_, queda muy limitada a la primera de las tres opciones. Véase:

(04a) ich kratze sie auf dem Rücken

(04d) !ich kratze sie hier, dort, da ${ }^{11}$

(04e) *ich kratze sie darauf

10 En el caso de kratzen, y algún otro como jucken, etc., constatamos la existencia de dos estructuras oracionales diferentes. La primera de ellas debe ser asociada al modelo V [NOM, AKK, DAT]: er kratzt sich den Bart > er kratzt seinen Bart, etc.; y la segunda lo es a este segundo modelo cuyo análisis abordamos en el presente capitulo V [NOM, AKK, ADV EST]. Exactamente lo mismo ocurre con el resto de verbos mencionados: jdn. an der Hand küssen > jdm. die Hand küssen, jdn. am Arm festhalten > jdm. die Hand festhalten, jdn. am Arm drücken $>$ jdm. den Arm drücken, jdn. am Arm streicheln $>$ jdm. den Arm streicheln, jdn. an der Hand verletzen > jdm. die Hand verletzen, etc. $11 \mathrm{El}$ ejemplo (04 d) y equivalente en (06) — no así en (5)— podrían mostrar gramaticalidad en determinados contextos en que la oración quedara reforzada con algún elemento paralingüístico (elementos prosódicos, lenguaje no verbal, etc.), por ejemplo de tipo deíctico. En un contexto de esas características - y es la razón por la que los hemos consignado de modo (') - sí sería plausible la aplicación de oraciones del tipo ich kratze mich hier (< auf dem Rücken), die Polizei packte ihn da, etc. 
Y exactamente del mismo modo en el caso de (05) y (06): er nimmt mich an der Hand $>$ *er nimmt mich hier $>{ }^{*}$ er nimmt mich daran, die Polizei packte ihn an der Kehle > 'die Polizei packte ihn da > *die Polizei packte ihn daran.

En tercer lugar, en todos estos casos es posible establecer un correlato o competencia respecto de las formas 'Genitivattribut' o 'adjektivisches Attribut'. Véase:

(04a) ich kratze sie auf dem Rücken

(04f) ich kratze auf ihrem Rücken

Y exactamente del mismo modo en el caso de (05) y (06): er nimmt mich an der Hand > er nimmt meine Hand, die Polizei packte ihn an der Kehle > die Polizei packte seine Kehle, etc. La opción (04f), y correspondiente en el resto de verbos seleccionados, se presenta como correlato semántico de (04 a), ello sin detrimento de las alteraciones pragmalingüísticas relacionadas con una focalización de la atención en (04 f) no tanto en la acción verbal como en la información contenida en el grupo nominal del constituyente ADV EST; bien en el valor de su componente nominal propiamente dicho ('Genitivattribut': ich kratze auf dem Rücken meiner Frau), o bien en la relación de pertenencia puesta de manifiesto por el posesivo en si ('adjektivisches Attribut': ich kratze auf ihrem Rücken) —en este caso, merced a las citadas alteraciones, podría marcarse de la siguiente manera: (04f) (i) ich kratze auf ihrem Rücken, (04f) (i) ich kratze auf dem Rücken meiner Frau-.

En el caso de este segundo modelo, y en lo relativo singularmente con la variante (04f), sería posible prescindir de la preposición de partida en favor de un régimen directo de 'Akkusativ'. Véase: (04a) ich kratze sie auf dem Rücken > (04f) ich kratze aufihrem Rücken / (04f) ich kratze ihren Rücken. Esta opción, inexistente en el modelo $1-(01$ a) er klopft ihm auf die Schulter > (01f) er klopft auf seine Schulter / (01f) * er klopft seine(r) Schulter - se hace obligatoria en el caso de algunos verbos de este segundo grupo, particularmente en todos aquellos cuyas bases introducen por principio un regimen de 'Akkusativ'; por ejemplo, los verbos nehmen o packen: (05a) er nimmt mich an der Hand > (05f) er nimmt meine Hand > *er nimmt an meiner Hand, o (06a) die Polizei packte ihn an der Kehle > (06f) die Polizei packte seine Kehle > (06f) *die Polizei packte an seiner Kehle. Sea como fuere, la omisión de la preposición no contraviene el carácter posesivo de las construcciones de partida, pues en ningún momento se prescinde del determinante posesivo 'sein'.

Finalmente, en cuarto lugar, debemos referirnos al carácter obligatorio del segundo constituyente $(\mathrm{AKK})$; esto es: el constituyente AKK podría ser omisible en el marco de cualquier otra relación 'no posesiva' (er kratzt an der Tür, etc.), pero no en el caso de mediar 'relación posesiva', pues en ese caso su supresión resultaría agramatical. Por su parte, el tercer constituyente resulta obligatorio sin restricciones. Véase: 
(04a) ich kratze sie am Arm

(04g) *ich kratze am Arm

(04h) *ich kratze sie ${ }^{12}$

Y exactamente del mismo modo er nimmt mich an der Hand $>{ }^{*}$ er nimmt an der Hand > 'er nimmt mich, o bien die Polizei packte ihn an der Kehle > * die Polizei packte an der Kehle > 'die Polizei packte ihn, etc. En el caso de las variantes marcadas como ('), y es la razón por la que las hemos marcado de esta manera, podría detectarse funcionamiento gramatical en el marco de usos desvinculados del ámbito posesivo, por ejemplo er nimmt mich mit nach Sevilla > er nimmt mich mit, o bien er packte das Geld > er packte es, etc., pero si se optara por algún ámbito posesivo relacionado con alguna 'parte del cuerpo' o 'prenda de vestir', dicho tercer constituyente sí tendría carácter obligatorio.

\section{El modelo oracional V [NOM, DAT, ADV EST]}

Hemos visto que los modelos V [NOM, DAT, ADV DIR] y V [NOM, AKK, ADV EST] alternan las formas 'Dativ' y 'Akkusativ', pues en aquel el segundo constituyente mostraba la forma 'Dativ' y el tercero la forma 'Akkusativ' como régimen prototípico de la preposición dinámica (recuérdese: er sieht mir in die Augen); y en este el segundo constituyente mostraba la forma 'Akkusativ' y el tercero la forma 'Dativ' como régimen prototípico de la preposición estática (recuérdese: er nimmt mich an der Hand). Las formas 'Dativ' y 'Akkusativ' se alternaban por consiguiente.

Existe un tercer modelo, sin embargo, en cuyo entorno los actantes 'segundo' y 'tercero', ambos, ahora sí, muestran una misma forma de DAT sobre la base de V [NOM, DAT, ADV EST]: en el caso del segundo constituyente por el propio régimen verbal y, en el caso del tercero por el carácter estático ADV EST relacionado con el significado verbal y la consiguiente aplicación de una preposición igualmente estática con 'Dativ'. Partamos, una vez más, de un pequeño corpus integrado nuevamente por tres ejemplos —véase según orden alfabético de la base verbal—:13
(07) brennen
(08) schmerzen
$>$ das Shampoo brennt mir in den Augen
(09) stechen
$>$ es schmerzt mir in der Hand
$>$ es sticht mir in den Augen

Una vez más, cualquiera de estos ejemplos muestra una clara rigidez formal en sus tres constituyentes; no solo del primero (NOM), sino también del segundo (DAT) y del tercero (ADV EST). Véase, así,

12 La oración (04h) podría funcionar con dos actates del tipo NOM kratzt AKK en caso de centrarse la información en la acción en sí sin referencia explícita ni implícita a ninguna parte del cuerpo en particular: beim Spielen haben sich die Kinder gekratzt. 13 Dejamos fuera de este análisis todas aquellas construcciones integradas igualmente por dos relaciones de DAT - al igual que en los casos (07), (08) o (09), la primera introducida directamente por el verbo y la segunda por la preposición- en que esta última, es decir, la relación introducida por la preposición, viene dada por un régimen obligatorio de dativo a partir de preposiciones del tipo aus, bei, zu, etc., en el marco de unidades de tipo tanto fraseolexemático (etwas steigt jdm. zu Kopf, jd. geht jdm. aus dem Weg, jd. geht jdm. aus den Augen, jd. liegt jdm. zu Füßen, etc.) como no fraseolexemático (etwas rutscht jdm. aus der Hand, etc.). 
la imposibilidad de alterar el segundo constituyente de DAT a AKK, o bien, en el caso del tercero, la imposibilidad de alterar el régimen preposicional del 'Dativ' propio de la relación ADV EST a 'Akkusativ':

(07a) das Shampoo brennt mir in den Augen

(07b) *das Shampoo brennt mich in den Augen

(07c) *das Shampoo brennt mir in die Augen

Y del mismo modo podemos proceder con (08) o (09): es schmerzt mir in der Hand $>$ * es schmerzt mich in der Hand $>$ * es schmerzt mir in die Hand, es sticht mir in den Augen > *es sticht mich in den Augen > * es sticht mir in die Augen, etc.

En segundo lugar, las limitaciones relativas a la morfología del constituyente ADV DIR afectan no solo al caso gramatical propiamente dicho sino a la categoría léxica, pues, en todos estos casos, una vez más, la habitual triple forma adverbial de 1) grupo preposicional con marcador direccional en 'Dativ', 2) adverbio propiamente dicho o 3) adverbio pronominal del tipo darauf, darüber, etc. —véase, una vez más, a partir de un verbo inlativo como sitzen, las correspondencias er sitzt auf dem Stuhl > er sitzt dort, o bien, a partir de ese mismo verbo, las correspondencias er sitzt auf dem Stuhl > er sitzt darauf -, queda muy limitada a la primera de las tres opciones. Véase:

(07a) das Shampoo brennt mir in den Augen

(07d) 'das Shampoo brennt mir dort ${ }^{14}$

(07e) *das Shampoo brennt mir darin

Véase igualmente: es schmerzt mir in der Hand > 'es schmerzt mir dort $>$ *es schmerzt mir darin, es sticht mir in den Augen > 'es sticht mir dort > *es sticht mir darin, etc.

En tercer lugar, en todos estos casos es posible establecer un correlato o competencia respecto de las formas 'Genitivattribut' o 'adjektivisches Attribut'. Véase:

(07a) das Shampoo brennt mir in den Augen

(07f) das Shampoo brennt in meinen Augen

Y exactamente del mismo modo en es schmerzt mir in der Hand $>$ es schmerzt in meiner Hand, es sticht mir in den Augen > es sticht in meinen Augen, etc. Una vez más, la opción (07f) y correspondiente en el resto de verbos seleccionados se presenta como correlato semántico de (07a), ello sin detrimento de las alteraciones pragmalingüísticas relacionadas con una focalización de la atención en (07f) no tanto en la acción verbal como en la información contenida en el grupo nominal del constituyente

14 También la oración (07d) y equivalentes en (08) y (09), y del mismo modo que ya indicábamos respecto de (04 d) y equivalentes en (05) y (6), podría mostrar gramaticalidad en determinados contextos en que la oración quedara reforzada con algún elemento paralingüístico (elementos prosódicos, lenguaje no verbal, etc.), por ejemplo de tipo deíctico. En un contexto de esas características -y es la razón por la que los hemos consignado de modo (')- sí sería plausible la aplicación de oraciones del tipo das Schampoobrennt mirhier (< in denAugen), etc. 
ADV EST; bien en el valor de su componente nominal propiamente dicho ('Genitivattribut': das Schampoo brennt in den Augen-meines Sohnes-), o bien en la relación de pertenencia puesta de manifiesto por el posesivo en sí ('adjektivisches Attribut': das Schampoo brennt in meinen Augen) —en este caso, por tanto, merced a las citadas alteraciones, podría marcarse de la siguiente manera: (07f) (") das Schampoo brennt in meinen Augen-.

Las constataciones establecidas en torno a la posibilidad de llevar a cabo pruebas de sustitución respecto de algún determinante posesivo, según resulta de comparar (07a) y (07f), sin embargo, no son aplicables a expresiones con carácter fraseolexemático dotadas igualmente de doble 'Dativ' del tipo jdm. auf den Fersen sein, etwas liegt jdm. im Magen, etwas liegt jdm. auf der Seele, etwas liegt jdm. am Herzen, jdm. auf der Nase herumtanzen, etc. Cualquiera de ellas, pese a responder igualmente al modelo establecido V [NOM, DAT, ADV EST], no obstante, por razón del citado carácter fraseolexemático y consiguiente inalterabilidad de sus partes, muestra mayores limitaciones al respecto, no solo en lo relativo a la posibilidad de dotar al constituyente ADV EST de alguna forma alternativa de adverbio o adverbio pronominal, algo que ya ocurría en el caso de las unidades 'no fraseolexemáticas' según pudimos ver en $(07 d)$ o $(07 e)$, sino, además en este caso, en lo relativo a la posibilidad de establecer competencias funcionales con la fórmula de determinante posesivo. Compárese el par (07a)-(07f) con cualquiera de estas otras unidades, por ejemplo jdm. auf den Fersen sein:

das Schampoo brennt mir in den Augen das Schampoo brennt in meinen Augen

er ist mir auf den Fersen [fraseolexema] *er ist auf meinen Fersen ${ }^{15}$ [fraseolexema]

Finalmente, en cuarto lugar, debemos referirnos al carácter obligatorio del segundo constituyente (DAT); esto es: el constituyente DAT podría ser omisible en el marco de cualquier otra relación 'no posesiva' (das Holz brennt im Garten, etc.), pero no en el caso de mediar 'relación posesiva', pues en ese caso su supresión resultaría agramatical. Por su parte, el tercer constituyente resulta obligatorio sin restricciones. Véase:

(07a) das Schampoo brennt ihm in den Augen

(07g) 'das Schampoo brennt in den Augen ${ }^{16}$

15 Los ejemplos fraseológicos que hemos indicado están vinculados a relaciones de dativo posesivo propiamente dicho, en los que está implicada una parte del cuerpo (Fersen en $j d$. ist jdm. auf den Fersen, etc.), pero la excepcionalidad de que son exponentes sería igualmente aplicable a otras estructuras igualmente fraseológicas no relacionadas con 'partes del cuerpo' como etwas steht jdm. im Wege (etwas steht mir im Weg > 'etwas steht in meinem Weg), etc.

16 Las oraciones (07g) y equivalentes en (08) y (09) solo podrian mostrar gramaticalidad en caso de referencia a algún 'Genitivobjekt' implícito: das Schampoo brennt in den Augen (des Kindes, etc.), etc., algo a lo que por otra parte ya aludimos al referirnos a la competencia respecto de las formas 'Genitivattribut' o 'adjektivisches Attribut' de este tercer modelo. Esta posible supresión del constituyente DAT nos permite atribuirle un estatus mixto: como dativo posesivo, algo que se ve corroborado por la existencia de alguna fórmula alternativa basada en la aplicación de algún determinante posesivo según ya ha quedado expuesto - recuérdese Das Shampoo brennt mir in den Augen > 
(07h) !das Schampoo brennt ihm ${ }^{17}$

Y exactamente del mismo modo es schmerzt mir in der Hand $>$ !es schmerzt in der Hand > 'es schmerzt mir, o bien es sticht mir in den Augen > 'es sticht in den Augen > 'es sticht mir, etc.

\section{El modelo oracional V [NOM, AKK, ADV DIR]}

Finalmente, también sería posible determinar un modelo del tipo V [NOM, AKK, ADV DIR], integrado por tanto por dos formas de 'Akkusativ': la correspondiente al segundo constituyente AKK por tratarse de una función de complemento directo y la introducida por la preposición - preposición del tipo 'Wechselpräposition', una vez más- dentro del entorno del tercer constituyente ADV DIR. Partamos, una vez más, de un pequeño corpus integrado nuevamente por tres ejemplos según orden alfabético de la base verbal:
(10) nehmen
$>$ er nimmt mich in die Arme
(11) beißen
$>$ der Hund beißt mich ins Bein
(12) hauen
$>$ der Mann haut mich in den Bauch

Una vez más, cualquiera de estos ejemplos muestra una clara rigidez formal en sus tres constituyentes; no solo del primero (NOM), sino también del segundo (AKK) y del tercero (ADV DIR). Véase, así, la imposibilidad de alterar el segundo constituyente de AKK a DAT, o bien, en el caso del tercero, la imposibilidad de alterar el régimen preposicional del 'Akkusativ' propio de la relación ADV DIR a 'Dativ':

(10a) er nimmt mich in die Arme

(10b) *er nimmt mir in die Arme

(10c) *er nimmt mich in den Armen

\footnotetext{
Das Shampoo brennt in meinen Augen-; pero, al tiempo, también como dativo libre, algo que se ve corroborado por la correlación existente entre (07g) y (07a). Naturalmente, este carácter omisible de DAT viene dado no tanto por el modelo en sí [NOM, DAT, ADV EST] de forma apriorística como por la propia concepción monovalente del significado de que es exponente la base verbal brennen (das Holz brennt, der Wald brennt, etc.), siendo, al tiempo, precisamente ese carácter optativo lo que nos permite otorgarle la antedicha consideración de dativo libre. En definitiva, en el caso de brennen, y también ocurre en el de otros verbos de este grupo (schmerzen, stechen, etc.), la activación del constituyente DAT y, con ello, la propia existencia del modelo en sí, viene dada no tanto por la disponibilidad previa de un modelo de estas características - del modo que ocurre con klopfen en jdm. auf die Schulter klopfen en relación con [NOM, DAT, ADV DIR], o bien con nehmen en jdn. an der Hand nehmen en relación con [NOM, AKK, ADV EST] - como por la consideración 'libre' del constituyente DAT.

17 Igualmente omisible es el constituyente ADV EST según apreciamos en (07 h), y también en sus equivalentes en (08) y (09), algo que, una vez más, está relacionado con el carácter prototipicamente monovalente de brennen, que hace de ADV EST un constituyente eventualmente omisible de tipo 'fakultative Angabe' - en el caso tanto del primer modelo [NOM, DAT, ADV EST] como del segundo modelo [NOM, AKK, ADV DIR], sin embargo, ni el segundo ni el tercer actante, además naturalmente del primero, eran omisibles, algo relacionado con el carácter prototípicamente trivalente o, al menos, bivalente de las correspondientes bases verbales-. Incluso, en estos casos (07), (08) y (09), y por las mismas razones expuestas anteriormente, cabría la posibilidad de omitir ambos, tanto el segundo (DAT) como el tercero (ADV EST). Véase brennen > es brennt (mir) (in den Augen), schmerzen > es schmerzt (mir) (in der Hand), stechen > es sticht (mir) (in den Augen).
} 
Y del mismo modo podemos proceder con (11) o (12): der Hund beißt mich ins Bein > 'der Hund beißt mir ins Bein > *der Hund beißt mich im Bein, der Mann haut mich in den Bauch > 'der Mann haut mir in den Bauch $>$ *der Mann haut mich in dem Bauch, etc. Algunos de estos verbos vinculados al modelo $\mathrm{V}$ [NOM, AKK, ADV DIR] admitirian una alteración del constituyente AKK a DAT con un consiguiente matiz semántico de no voluntariedad en la acción. Compárese así la alteración de significado existente entre los citados der Hund beißst mich ins Bein y der Hund beißt mir ins Bein, der Mann haut mich in den Bauch y der Mann haut mir in den Bauch, o cualesquiera otros: der Mann sticht mich in den Arm y der Mann sticht mir in den Arm, etc. Para esta distinción véase Mellado (2006), quien, al abordar este asunto, se refiere al interesante ejemplo der Rauch beißt mir in die Augen, el cual, al venir mercado por un sujeto (der Rauch) no personal y que, por tanto, no asume voluntariedad, no permitiría el cambio a AKK. Véase: der Rauch beißt mir in die Augen > *der Rauch beißt mich in die Augen.

En segundo lugar, las limitaciones relativas a la morfología del constituyente ADV DIR afectan no solo al caso gramatical propiamente dicho sino a la categoría léxica, pues, en todos estos casos, la habitual triple forma adverbial de 1) grupo preposicional con marcador direccional en 'Akkusativ', 2) adverbio propiamente dicho o 3) adverbio pronominal del tipo darauf, darüber, etc. —véase, a partir de un verbo adlativo como fliegen, las correspondencias er fliegt auf die Insel > er fliegt dorthin, o bien, a partir de un verbo igualmente adlativo como schreiben, las correspondencias er schreibt auf das Papier > er schreibt darauf -, vuelve a quedar muy limitada a la primera de las tres opciones. Véase:

(10a) er nimmt mich in die Arme

(10d) *er nimmt mich dorthin

(10e) *er nimmt mich darin

Y exactamente del mismo modo: der Hund beißt mich ins Bein $>*$ der Hund beißt mich dorthin $>*$ der Hund beißt mich darin, der Mann haut mich in den Bauch $>*$ der Mann haut mich dorthin $>*$ der Mann haut mich darein, etc.

En tercer lugar, en todos estos casos es posible establecer un correlato o competencia respecto de las formas 'Genitivattribut' o 'adjektivisches Attribut'. Véase:

er nimmt mich in die Arme

er nimmt mich in seine Arme ${ }^{18}$

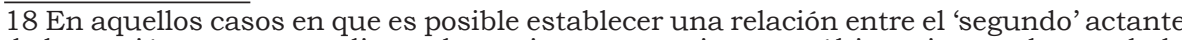
de la oración y correspondiente determinante posesivo, este último siempre ha quedado referido a la persona de la que era exponente el actante propiamente dicho. Recuérdese el par er sieht mir in die Augen > er sieht in meine Augen en relación con el Modelo V [NOM, DAT, ADV DIR], en cuyo ámbito el constituyente mir compite con el posesivo meine, razón por la que este queda marcado con la persona de la que aquel es exponente (mich > meine) y no con otra, etc. En el caso de este cuarto modelo V [NOM, AKK, ADV DIR] debemos distinguir entre aquellos casos en que la 'parte del cuerpo' queda vinculada al constituyente NOM, por ejemplo Arme en er nimmt mich in die Arme (el constituyente Arme pertenece a la persona representada por NOM) y aquellos otros en que lo hace al constituyente AKK, por ejemplo der Hund beißt mich ins Bein (el constituyente Bein pertenece a la persona representada por AKK). En este último caso la estructura 
Y exactamente del mismo modo der Hund beißt mich ins Bein > der Hund beißt in mein Bein, der Mann haut mich in den Bauch > der Mann haut in meinen Bauch, etc. La opción (10f), y correspondiente en el resto de verbos seleccionados, se presenta como correlato semántico de (10a), ello sin detrimento de las alteraciones pragmalingüísticas relacionadas con una focalización de la atención en (10f) no tanto en la acción verbal como en la información contenida en el grupo nominal del constituyente ADV DIR; bien en el valor de su componente nominal propiamente dicho ('Genitivattribut': der Mann haut in den Bauch seines Feides), o bien en la relación de pertenencia puesta de manifiesto por el posesivo en sí ('adjektivisches Attribut': der Mann haut in seinen Bauch) —en este caso, merced a las citadas alteraciones, podría marcarse de la siguiente manera: (10f) (!) der Mann haut in den Bauch seines Feides, (10f) (') der Mann haut in seinen Bauch-.

Las constataciones establecidas en torno a la posibilidad de llevar a cabo pruebas de sustitución respecto de algún determinante posesivo, según resulta de comparar (01a) y (01f), sin embargo, no son aplicables a expresiones con carácter fraseolexemático del tipo jdn. auf den Arm nehmen. Cualquier estructura de este tipo, pese a responder igualmente al modelo establecido V [NOM, AKK, ADV DIR], no obstante, merced al citado carácter fraseolexemático y consiguiente inalterabilidad de sus partes, muestra mayores limitaciones al respecto, no solo en lo relativo a la posibilidad de dotar al constituyente ADV DIR de alguna forma alternativa de adverbio o adverbio pronominal, algo que ya ocurría en el caso de las unidades 'no fraseolexemáticas' según pudimos ver en (10d) o (10e), sino, además en este caso, en lo relativo a la posibilidad de establecer competencias funcionales con la fórmula de determinante posesivo. Compárese el par (10a)-(10f) con cualquiera de estas otras unidades, por ejemplo jdn. auf den Arm nehmen:

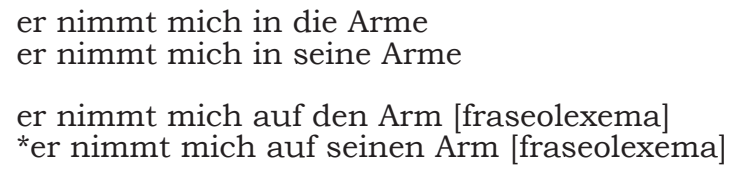

Finalmente, en cuarto lugar, debemos referirnos al carácter obligatorio del segundo constituyente (AKK); esto es: el constituyente AKK podría ser eventualmente omisible en el marco de relaciones 'no posesivas' (er nimmt, er beißst, er haut, etc.), pero no en el caso de mediar 'relación posesiva', pues en ese caso su supresión resultaría agramatical. Por su parte, el tercer constituyente resulta obligatorio sin restricciones. Véase:

(01a) er nimmt mich in die Arme

(01g) *er nimmt in die Arme

(01h) *er nimmt mich

alternativa mediante 'Genitivattribut' o ‘adjektivisches Attribut' pasa por la desaparición del constituyente AKK (der Hund beißt mich ins Bein > *der Hund beißt mich in mein Bein), mientras que en aquel primer caso es necesaria, de una parte su permanencia, $\mathrm{y}$, de otra, la referencia del determinante posesivo no tanto a AKK como a NOM (véase er nimmt mich in die Arme > *er nimmt in meine Arme > er nimmt mich in seine Arme). 
Y exactamente del mismo modo der Hund beißt mich ins Bein $>$ !der Hund beißt ins Bein > *der Hund beißt mich, o bien der Mann haut mich in den Bauch > 'der Mann haut in den Bauch > *der Mann haut mich. ${ }^{19}$

\section{Conclusiones}

1.- Partiendo de la estructura básica de 'Dativo posesivo' V [NOM, DAT, ADV DIR] con referencia a alguna 'parte del cuerpo' en cuyo ámbito los constituyentes NOM y DAT muestran forma de grupo nominal (NP) y el constituyente ADV DIR forma de grupo preposicional (PP), sería posible extrapolar esta base a tres modelos adicionales con una misma distribución actancial (NP, NP, PP) - siempre dentro de un entorno 'posesivo' con referenciación de alguna 'parte del cuerpo'-, en cuyo ámbito los constituyentes segundo y tercero alternarian las formas de 'Dativ' y 'Akkusativ', en virtud de las siguientes combinaciones: V [NOM, DAT, ADV DIR], V [NOM, AKK, ADV EST], V [NOM, DAT, ADV EST] y V [NOM, AKK, ADV DIR].

2.- Tomando como punto de partida una inmutabilidad de constituyentes propia de cualquiera de los verbos escogidos en nuestro muestrario, constatamos las limitaciones que todos ellos muestran respecto de posibles alteraciones en el constituyente adverbial ADV a formas alternativas a la ya mencionada de grupo preposicional, por ejemplo adverbios pronominales o adverbios propiamente dichos; e, igualmente, la tendencia de los cuatro modelos a admitir aplicaciones alternativas basadas en el uso de determinantes posesivos, ello sin detrimento de las posibles matizaciones de significado que pueda acarrear una u otra fórmula.

3. La relación posesiva, por tanto, no se circunscribe al modelo $\mathrm{V}$ [NOM, DAT, ADV DIR] que suele tomarse como paradigmático a la hora de hablar de un 'Dativo posesivo' —véanse, una vez más, ejemplos del tipo er klopft mir auf die Schulter, etc. y también similares dentro del entorno 'Träger-Dativ' al que aludiamos al comienzo: ich ziehe mir den Mantel an, etc. - sino que es igualmente aplicable a los modelos V [NOM, AKK, ADV EST], V [NOM, DAT, ADV EST] y $\mathrm{V}$ [NOM, AKK, ADV DIR]. En el caso de los modelos V [NOM, DAT, ADV DIR] y $\mathrm{V}$ [NOM, DAT, ADV EST] vendrá dada por el constituyente en 'Dativ' y en el modelo V [NOM, AKK, ADV EST] y V [NOM, AKK, ADV DIR] lo hará por el constituyente 'Akkusativ'. Los cuatro modelos, por consiguiente, tanto los dos marcados por 'Dativ' como los dos marcados por 'Akkusativ', muestran una relación igualmente posesiva respecto de los referentes 'partes del cuerpo' a que se remiten, de tal modo que cabria estandarizar una relación posesiva no solo de 'Dativ' mediante el denominada 'Dativo Posesivo' sino también de 'Akkusativ' mediante un, ¿por qué no?, 'Acusativo Posesivo'.

19 Las opciones sin constituyente adverbial podrían funcionar en caso de centrarse la información en la acción en sí, sin referencia explícita ni implícita a ninguna parte del cuerpo en particular: der Hund beißt mich, der Mann haut mich, etc. 


\section{REFERENCIAS}

ADMONI, W. (1982): Der deutsche Sprachbau, Múnich: Beck'sche Elementarbücher.

DROSDOWSKI, G. (1995): Duden Grammatik der deutschen Gegenwartssprache, Mannheim: Duden.

ENGEL, U. \& SCHUMACHER, H. (1976): Kleines Valenzlexikon deutscher Verben, Tubinga: Gunter Narr.

HELBIG, G. \& BUSCHA, J. (1996): Deutsche Grammatik, Berlin: Langenscheidt.

HELBIG, G. \& SCHENKEL, W. (1969): Wörterbuch zur Valenz und Distribution deutscher Verben, Berlin: de Gruyter.

HOCKE, M. (1987): Die Pertinenzrelation im Deutschen, Frankfurt: Peter Lang.

LÓPEZ-CAMPOS BODINEAU, R. (1993): "Consideraciones semánticas acerca del uso del dativo alemán y sus posibles concurrencias con otro tipo de construcciones", Philologia Hispalensis, 8, pp. 245-258.
MELLADO BLANCO, C. (2006): "El dativo posesivo en alemán en contraste con otras lenguas europeas", Estudios Filológicos Alemanes, 11, pp. 177-194.

MELLADO BLANCO, C. (2012): "Zur syntaktisch-semantischen Charakterisierung des Pertinenzdativs im Deutschen und Spanischen", Sprachtheorie und germanistische Linguistik, 22(9): "Der Pertinenzdativ und seine Satzbaupläne", Festschrift für Hugo Moser, Düsseldorf: Schwann, pp. 146-171.

SCHÄFER, R. (2015): Einführung in die grammatische Beschreibung des Deutschen, Berlin: Language Science Press. SCHMID, J. (2006): "Die freien Dative", Dependenz und Valenz (Band 2), Berlin: de Gruyter. 